\title{
Desafíos para la Transposición Didáctica y Conocimiento Didáctico del Contenido en Docentes de Anatomía: Obstáculos y Proyecciones
}

\author{
Challenges to the Didactic Transposition and Knowledge \\ of the Content in Teaching of Anatomy: Obstacles and Projections
}

\author{
"Mario Pellón Arcaya; **Juan Mansilla Sepúlveda \& ***Daniel San Martín Cantero
}

\begin{abstract}
PELLÓN, A. M.; MANSILlA, S. J. \& SAN MARTíN, C. D. Desafíos para la transposición didáctica y conocimiento didáctico del contenido en docentes de anatomía: obstáculos y proyecciones. Int. J. Morphol., 27(3):743-750, 2009.

RESUMEN: La enseñanza de la Anatomía a nivel de pre-grado exige una formación basada en competencias que genere resultados de aprendizajes, donde converjan saberes sabios de la disciplina con saberes didácticos de la pedagogía. Para ello, se planteó un diseño cualitativo de investigación con el propósito de describir desafíos, obstáculos y proyecciones de la transposición didáctica y el conocimiento didáctico del contenido disciplinario de la Anatomía en la Escuela de Medicina de la Universidad Mayor de Temuco, en base a la Teoría Fundamentada, Método Comparativo Constante y Triangulación Metodológica. La muestra se constituyó con la totalidad de los docentes de la cátedra de Anatomía. A partir de la codificación abierta emergieron cinco categorías: a) dificultades del ejercicio docente, b) recursos materiales y humanos, c) planificación y estrategias de evaluación, d) estrategias de enseñanza-aprendizaje y e) requerimientos y características. Las técnicas de recolección de información fueron: observación etnográfica, entrevista semiestructurada y grupos focales. Para el procesamiento de data verbal se utilizó el software para el análisis cualitativo de datos Atlas-ti versión 5.0. Los resultados del estudio develan incoherencias de carácter epistémico en la actuación didáctica de los docentes, quienes sitúan su práctica desde la lógica tradicionalista experiencial, apartándose de métodos dialógicos dialécticos necesarios en la actual cartografía sociocultural de la que forman parte los estudiantes de Pre-grado.
\end{abstract}

PALABRAS CLAVE: Transposición didáctica; Enseñanza de la Anatomía; Conocimiento disciplinario; Investigación cualitativa.

\section{INTRODUCCIÓN}

Actualmente, la formación de profesionales en la Educación Superior desafía a los docentes a incorporar en sus prácticas de enseñanza no solamente el dominio de contenido disciplinario puro, sino que además les exige competencias asociadas a la apropiación de conocimiento didáctico para vehiculizar el saber disciplinario hacia el saber pedagógico, con el objeto de cumplir con el acto educativo.

Lo anterior implica ciertas transformaciones en las prácticas pedagógicas de la docencia universitaria, lo cual es una problemática particularmente importante en la ense- ñanza de contenidos disciplinarios en las ciencias de la salud, donde convergen actividades teóricas, prácticas e integradas que complejizan la enseñanza.

Todo cambio lleva exigencias. Frente a los posicionamientos clásicos centrados en el aula y la actividad del profesor hoy se propugna una enseñanza centrada sobre la actividad autónoma del alumno, lo que significa cambios en la planificación de currícular en tanto organización de la enseñanza. Lo anterior no implica distribuir linealmente los contenidos a lo largo de un programa, sino de autorizar

* Médico Cirujano Oftalmólogo. Magíster en Pedagogía Universitaria. Docente Facultad de Medicina. Universidad de La Frontera y Universidad Mayor. mpellon@ufro.cl. Casilla 54-D. Temuco, Chile.

** Profesor de Estado. Magíster en Desarrollo Regional y Local. Docente Postgrado Universidad Mayor y Universidad Católica de Temuco. jmansilla@ uct.cl. Casilla 15 -D, Temuco, Chile.

**** Profesor de Estado. Docente Universidad Católica de Temuco. dsanmartin@uct.cl. Casilla 15 -D. Temuco, Chile. 
secuencialmente las experiencias de aprendizaje de los estudiantes, guiando sus aprendizajes (De Miguel-Díaz, 2005).

Estas transformaciones tensionan a los paradigmas tradicionales de enseñanza y generan crisis, lo que desde las tesis de Khun implican una revolución científica, y por lo tanto, una cambio paradigmático (Khun, 1982).

En este contexto de yuxtaposición entre la tradición pedagógica y la innovación curricular, surge la propuesta del Conocimiento Pedagógico del Contenido cuyo origen está en el denominado Conocimiento Didáctico del Contenido (CDC), el cual se remonta a una conferencia que Lee Shulman pronunció en la Universidad de Texas -en Austindurante el verano de 1983, titulada: "El paradigma perdido en la investigación sobre la enseñanza" (Shulman, 2005). Este paradigma resultó ser "el pensamiento del profesor sobre el contenido del tema objeto de estudio y su interacción con la didáctica”.

Según Shulman, el conocimiento base para la enseñanza de un profesor debe incluir al menos siete categorías de conocimiento diferentes: (i) conocimiento del contenido, (ii) conocimiento didáctico general, (iii) conocimiento curricular, (iv) conocimiento didáctico del contenido, (v) conocimiento de las características, los aspectos cognitivos, la motivación de los estudiantes, (vi) conocimiento de los contextos educativos y (vii) conocimiento de las finalidades educativas, los valores educativos y los objetivos. (Acevedo, 2009).

Estos siete tipos de conocimiento base fueron redifinidos posteriormente por Grossman (2005) en cuatro grupos más generales: (i) conocimiento didáctico general, (ii) conocimiento del contenido, (iii) conocimiento didáctico del contenido y (iv) conocimiento del contexto. Según algunos autores, una noción más inclusiva del CDC resulta mejor que una demasiado extensa (Acevedo).

El interés por el CDC se debe, sobre todo, a que implica un conjunto de saberes que permite al profesor trasladar a la enseñanza el contenido de un determinado tópico; esto es, hacer la transposición didáctica del conocimiento especializado de un tema al conocimiento escolar objeto de enseñanza y aprendizaje (Acevedo).

Es decir, el CDC es una combinación adecuada entre el conocimiento de la materia a enseñar y el conocimiento pedagógico y didáctico relativo a cómo enseñarla. Asimismo, incluye en el CDC:

“[...] las formas más útiles de representación [...], analogías, ilustraciones, ejemplos, explicaciones y demostracio- nes, es decir, las formas de representar y formular el tema que lo hacen comprensible a los otros [...] además de la comprensión de lo que hace fácil o difícil el aprendizaje de un tema concreto: Las concepciones e ideas previas que los estudiantes de diferentes edades traen al aprendizaje." (Shulman)

El modelo integrador de CDC de Gess-Newsome (1999) considera tipos de conocimiento que se constituyen en conceptos claves para yuxtaponer los saberes disciplinarios, didácticos y contextuales, complejizando la actuación docente, donde hay un desafío a la superación de una enseñanza tradicional magistocéntrica, de tal forma que ésta se oriente a una lógica paidocéntrica ( Fig. 1).

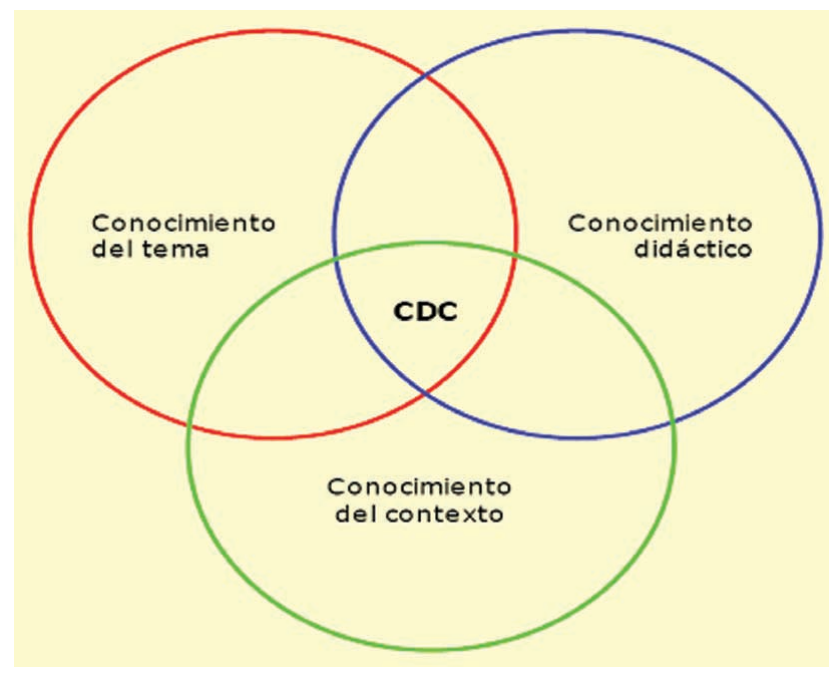

Fig. 1. Modelo integrador del CDC según Gess-Newsome (1999). Tomado y modificado de Acevedo. Conocimiento didáctico para el contenido de la enseñanza de la ciencia (I): El marco teórico, 2009.

Chevallard (1985) definió la transposición didáctica como el trabajo que transforma el objeto de saber en un objeto de enseñanza. El sujeto va adquiriendo conocimientos de su mundo en la medida que es capaz de ir captando aquellas propiedades que lo caracterizan. A partir de esta premisa la fuente psicológica de la didáctica radica, por una parte, en encontrar los mecanismos que faciliten a los educandos la adquisición de las propiedades y características del objeto disciplinar estudiado, como así también determinar la etapa de desarrollo cognitivo en la que los sujetos están capacitados intelectualmente para aprender objetos de enseñanza específicos (Díaz, 2003).

El trabajo de transformación al que alude Chevallard, aquí es entendido como el cambio que experimenta el objeto de saber al ser reconstruido en el aula de manera intersubjetiva entre el profesor que domina los conocimien- 
tos de su disciplina y sus alumnos, quienes ya cuentan con un saber experiencial. El objeto de conocimiento resultante, es decir el objeto de enseñanza, ya no es exactamente el mismo del cual que se origina, pero mantiene las cualidades que lo distinguen como tal y que permiten validarlo intersubjetivamente por aquellos sujetos del ámbito educativo que lo reconstruyeron (Chevallard).

Por consiguiente, la labor de la didáctica involucra necesariamente reconocer, por una parte, las peculiaridades culturales de la disciplina desde donde se origina un objeto de saber entendidas como la lógica disciplinar, y por otra, distinguir las peculiaridades de la cultura escolar en donde se efectuará la transposición didáctica de dicho objeto de saber en objeto de enseñanza, entendida como lógica de la enseñanza y lógica del aprendizaje.

Respecto de estas evidencias empíricas y discusión teórica surge el siguiente problema de investigación: ¿Cuáles son los componentes didácticos que son utilizados actualmente por los docentes en sus prácticas de enseñanza de Anatomía en la Escuela de Medicina de la Universidad Mayor de Temuco?

El objetivo de esta investigación fue describir el uso de las estrategias didácticas metodológicas utilizadas en la enseñanza de Anatomía para estudiantes de Medicina. A partir de los resultados de la investigación se propone revisar las prácticas pedagógicas de los docentes respecto de la enseñanza de contenidos disciplinarios, con el objeto de generar reflexión de la acción educativa en la enseñanza de la Anatomía.

\section{METODOLOGÍA}

El método de investigación es cualitativo descriptivo hermeneútico porque tiene relevancia específica para el estudio de las relaciones sociales, debido al hecho de la pluralización de los mundos vitales (Flick, 2004). La muestra fue intencional y está constituida por la totalidad de los docentes de la cátedra de Anatomía de la Escuela de Medicina de la Universidad Mayor, quienes imparten docencia a 70 estudiantes de primer año. Los instrumentos de recolección de datos fueron entrevistas semiestructuradas, grupos focales y observaciones etnográficas.

Los grupos focales permiten al investigador "alinearse con los participantes y descubrir cómo ven la realidad". A su vez, la entrevista permite que se manifiesten espontáneamente los significados y jerarquías de valores del entrevistado.
En la etapa de "saturación teórica" de desarrolló el muestreo teórico donde los datos se tornaron repetitivos. Flick define el criterio de Saturación Teórica como: “(...) el muestreo e integración de material nuevo se acaba cuando la "saturación teórica" de una categoría o grupo de casos se ha alcanzado, es decir, cuando no emerge ya nada nuevo"

Al respecto Strauss \& Corbin (2002) señalan que el criterio que asegura la riqueza y densidad explicativa, y de relaciones teóricas entre categorías es la saturación teórica de éstas, por esto el único criterio para finalizar el proceso de recolección de datos es la saturación de los mismos, lo cual finalmente explicará la variabilidad de las categorías.

Para efectos de esta investigación se realizó una codificación abierta al proceso, en el que el investigador aborda el texto, con el fin de develar los conceptos, ideas y sentidos, que éste contiene. Respecto a esto, Strauss \& Corbin son claros señalando "que para descubrir y desarrollar los conceptos debemos abrir el texto y exponer los pensamientos, ideas y significados contenidos en él" (Strauss \& Corbin). Durante este proceso de Codificación Abierta los datos fueron descompuestos, examinados y comparados en términos de sus similitudes y diferencias, al amparo de una aproximación inductiva a la realidad, por lo que no precisa de una teoría para aplicar sus conceptos, leyes o dimensiones en el texto que se está codificando, por el contrario, el investigador debe zambullirse en el texto para así identificar los conceptos, dimensiones y construir categorías y subcategorías pertinentes a la investigación.

\section{RESULTADOS}

Una vez realizada la triangulación metodológica de datos a partir de los códigos levantados, surgieron cinco categorías: (1) Dificultades ejercicio docente (subcategorías debilidades del desempeño docente, debilidades del medio, y debilidades asociadas a la gestión directiva); (2) recursos materiales y humanos (subcategorías recursos materiales y recursos humanos); (3) planificación y estrategias de evaluación (sin subcategorías); (4) estrategias de enseñanzaaprendizaje (sin subcategorías); (5) requerimientos y características asignatura de Anatomía (subcategorías características Asignatura de Anatomía y requerimientos Asignatura de Anatomía) (Fig. 2).

\section{Presentación de las categorías y subcategorías}

\section{Categoría 1. Dificultades en el ejercicio docente:}

Subcategoría a. Debilidad del desempeño docente. En esta 


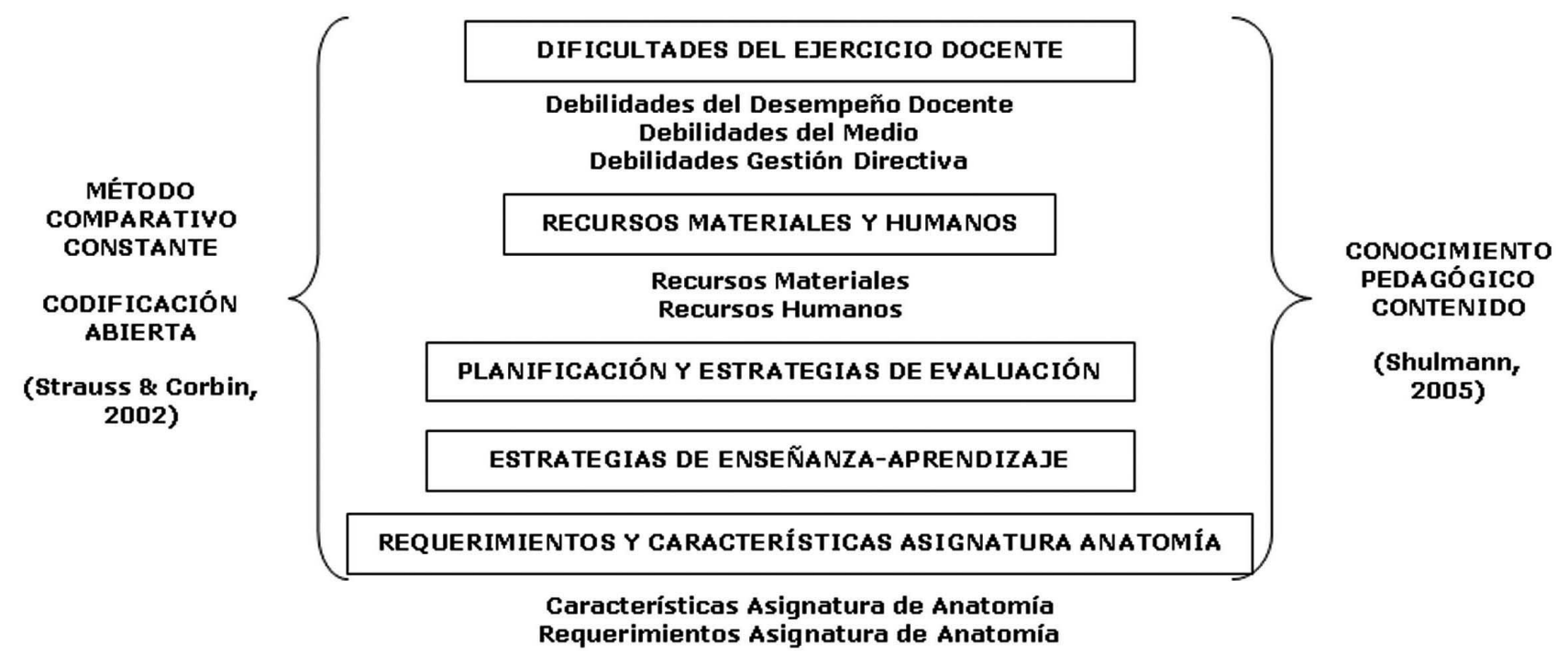

Fig. 2. Esquema teórico-metodológico de categorías y subcategorías obtenidas.

subcategoría destaca el rol docente en el desarrollo de la enseñanza, en tanto persisten representaciones de los docentes asociadas a una confusión de las acciones que se deben movilizar en el acto de enseñanza a partir de su rol en aula, por lo tanto, ellos exigen explicitar los rasgos de la labor pedagógica que son de su estricta responsabilidad. Esto, entonces, implica resituar el rol docente, desde la gestión que administra la enseñanza para el curso de Anatomía.

Subcategoría b. Debilidad del medio. En esta subcategoría el aspecto principal que aparece en el discurso de los informantes es el tiempo, y éste afecta procesos técnicos administrativos microcurriculares. La secuenciación y temporalización de la enseñanza no están implementadas adecuadamente. Esto se manifiesta, por una parte, en la argumentación/justificación de los docentes respecto de la imposibilidad para generar clases participativas debido a que éstas minimizan tiempo a la realización de clases tradicionales, donde se entregan contenidos disciplinarios; por otra parte, se evidencia una enseñanza centrada en la transmisión de la información, valorizando la cantidad de ésta que debe ser entregada, lo que obstaculiza disponer de tiempo para desarrollar competencias complejas en los estudiantes; y por último, aparece en el discurso de los docentes la percepción que no hay tiempos para llevar efectivamente a cabo prácticas de coordinación entre docentes para el diseño de enseñanza.

Subcategoría c. Debilidad de la gestión directiva. En esta subcategoría destaca principalmente la percepción de los docentes asociada a la ausencia de espacios formales y reales para dialogar, con el propósito de generar acuerdos pedagógicos didácticos, para generar resultados de aprendiza- jes en los estudiantes. Además, se explicita que existe interés en reflexionar sobre sus propias prácticas de enseñanza, con el objeto de tomar decisiones asertivas respecto de los métodos didácticos más pertinentes.

\section{Categoría 2. Recursos materiales y humanos:}

Subcategoría a. Recursos materiales. En esta subcategoría aparece la incorporación del data show, notebook y punteros láser con la finalidad de exponer imágenes referidas a objetos disciplinarios de la Anatomía (conceptos, relaciones, funcionamiento, entre otros). El uso de estos artefactos se usa durante toda la clase (inicio, desarrollo y cierre). A su vez, la usabilidad de la pizarra es esporádica y se limita al acto de improvisar dibujos aclaratorias de las diapositivas presentadas.

Subcategoría b. Recursos humanos. En esta subcategoría destaca la presencia de varios profesionales en la sala de clases con el propósito de profundizar conceptos basales de la clase para complementar la exposición disciplinaria del docente a cargo de la clase.

\section{Categoría 3. Planificación y estrategias de evaluación:}

Esta categoría no presentó subcategorías, sino que aparecen rasgos aislados desasociados y que se refieren a la planificación y estrategias de evaluación. En este sentido la cuestión más importante se refiere a la planificación individual de la enseñanza. Los informantes claves relevan la situación donde las prácticas de planificación de la enseñanza no responde a un diseño global para la asignatura desde la institucionalidad, más bien su elaboración responde a crite- 
rios personales, experienciales e intuitivos para organizar el desarrollo de la enseñanza. Lo anterior se respalda en tanto, los entrevistados reconocen no explicitar la preparación de la enseñanza en documentos, sino que se explicita en la oralidad.

\section{Categoría 4. Estrategias de enseñanza aprendizaje:}

Esta categoría no presentó subcategorías. En esta dimensión destaca la generación de preguntas abiertas desde el docente hacia los estudiantes. A partir de los registros etnográficos sistematizados se observa que una vez declarada la apelación, los estudiantes permanecen en silencio, entonces, el docente responde lo que él mismo ha preguntado. Asimismo, los registros evidencian, que los estudiantes no levantan preguntas en el desarrollo de la clase.

\section{Categoría 5. Requerimientos y características asignatura} de Anatomía:

Esta categoría contiene percepciones asociadas a necesidades de la Asignatura de Anatomía y algunas características implicadas en la enseñanza de ésta. En consecuencia, se develan dos subcategorías: 1) Características de Asignatura de Anatomía que contempla aspectos como la enseñanza tradicional, las clases que son de modalidad teórica práctica, la formación actitudinal de estudiantes y finalmente, la práctica vista como proyección de futuro profesional; 2) Requerimientos Asignatura Anatomía se evidencia una propuesta para la enseñanza, requerimientos del proceso de enseñanza-aprendizaje, también los informantes señalan que es importante el autoaprendizaje de los alumnos, finalmente se establece que se requiere una preparación de las clases.

Subcategoría 1. Características asignatura de Anatomía. Tradicionalmente la enseñanza de la Anatomía se desarrollar desde enfoques clásicos de transmisión de los saberes disciplinarios desde un posicionamiento de docente experto a un estudiante novato en esta materia. Las entrevistas evidencian que la asignatura de anatomía tiene como característica principal la enseñanza tradicional de los contenidos. Esta condición de la asignatura, desde la percepción de los docentes entrevistados, se extiende a otras Universidades que imparte esta asignatura.

Respecto de la subcategoría características de la Asignatura de Anatomía se levantaron los siguientes códigos: clases teórica-práctica, enseñanza tradicional, equipo de trabajo docente en sala. Las clases teóricas prácticas indican que la actividad de enseñanza es compleja, lo que implica que las clases están fundamentadas en dimensiones teóricas, pero a la vez se basan en demostraciones prácticas. A su vez la enseñanza tradicional implica que es el docente quien posee el saber sabio y que los transmite a los estudiantes, quienes se acercan al conocimiento disciplinario a partir del la información que proporciona el docente. Otro código es el trabajo colaborativo de otros profesionales en sala, lo que implica que la actividad pedagógica se complementa con otros profesionales vinculados a este saber disciplinario.

Subcategoría 2. Requerimientos. En esta subcategoría aparece declarado en el discurso de los docentes la necesidad de desarrollar en los estudiantes competencias personales asociadas a la autonomía con el objeto de autorregular aprendizajes, para que en las clases manifiesten conocimiento disciplinar de los contenidos. Este desafío requiere la mediación pedagógica del docente para orientar a los estudiantes en el logro de la competencia vinculada a la autonomía.

Respecto de la subcategoría requerimientos se evidenciaron lo siguientes códigos: autoaprendizaje, propuesta de enseñanza e internalización de conceptos descriptivos. El autoaprendizaje se percibe como una necesidad de la asignatura para con los estudiantes, para así lograr las competencias en la gestión de su propio aprendizaje y de su formación. A su vez, la propuesta de enseñanza está asociada a la necesidad que desde la coordinación de la gestión de la enseñanza de la Anatomía existan criterios definidos para abordar sinergéticamente esta compleja formación. Finalmente, la internalización de conceptos descriptivos implica que no sólo basta con el aprendizaje reproductivo, sino que implica avanzar hacia aprendizajes expansivos (learning by expanding) que movilicen el saber en todas sus dimensiones.

Las subcategorías explicitadas, además se asocian a la importancia que representa la internalización de conceptos por parte de estudiantes, pues esto es una característica y asimismo corresponde a un requerimiento de la Asignatura de Anatomía.

\section{DISCUSIÓN}

En el estudio realizado, cuyo objetivo general describir desafíos, obstáculos y proyecciones de la transposición didáctica y el conocimiento didáctico del contenido disciplinario de la Anatomía en la Escuela de Medicina de la Universidad Mayor de Temuco, es posible señalar, a modo general, que no existen prácticas asociadas a metodologías planificadas. Las prácticas de los docentes no constituyen una labor pedagógica sistemática ni enmarcada en paradigmas actuales de la educación, lo que conlleva a que la enseñanza sea tradicionalista y no responda eficientemente a las exigencias curriculares estipuladas por el nivel de coordinación, esto derivado de las evidencias expuestas. 


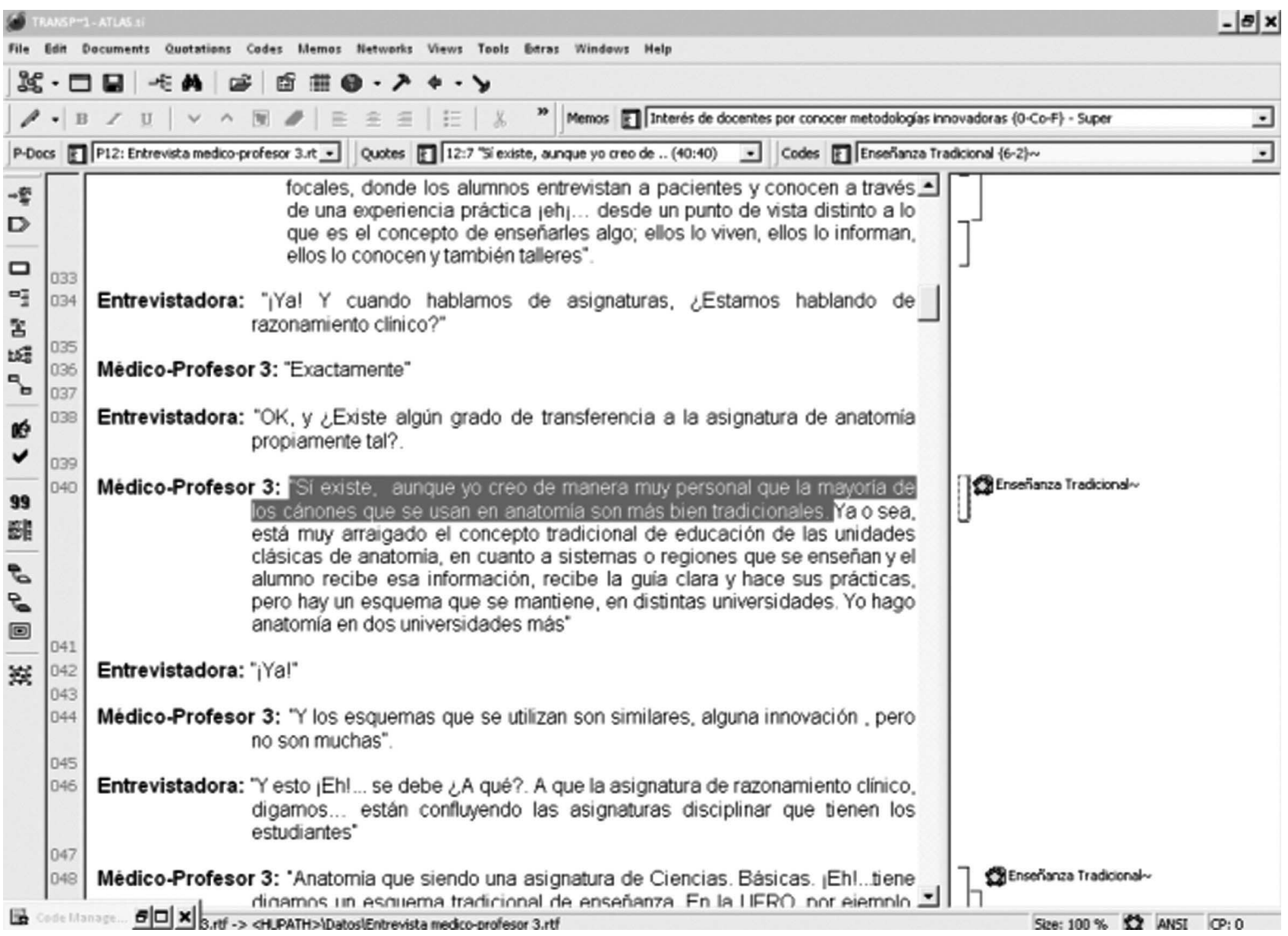

Fig. 3. Análisis de datos correspondientes a la categoría requerimientos y características Asignatura de Anatomía.

De acuerdo al segundo objetivo general: determinar el grado de conformidad de los docentes con sus prácticas de enseñanza, es posible afirmar que se aprecia conformidad con el desempeño tradicional de la enseñanza, aún cuando incorporan en sus prácticas ciertos recursos tecnológicos. Por tanto, concretamente no se innova debido a múltiples razones contextuales, pero la más importante dice relación con el carácter de la Asignatura de Anatomía enmarcada en la tradición, la falta de consenso acerca de estrategias de enseñanza-aprendizaje y la variable tiempo. Lo anterior deriva en una creencia básica de los docentes de Anatomía expresando que ésta es una disciplina descriptiva, por tanto la enseñanza no se concibe desde otro enfoque. Formalmente, esto corresponde a que, frecuentemente, los profesores tratan sus creencias como conocimiento, entonces, el dilema conocimiento de la materia y acción didáctica (Grossman et al.) están presentes en los docentes estudiados, de manera que las creencias afectan poderosamente a sus prácticas de enseñanza.

Los principales hallazgos indican que no es posible apreciar estrategias didácticas planificadas para abordar los contenidos disciplinarios. Las estrategias no estimulan el autoaprendizaje porque las prácticas están centradas en el profesor y no en el estudiante como constructor de su propio aprendizaje. En este sentido (De Miguel) señala que para propiciar un cambio de paradigma de la enseñanza-aprendizaje, es fundamental que el profesor facilite las herramientas necesarias para que el estudiante lleve a cabo la búsqueda personal hacia la mejora de su desempeño como estudiante, cuestión que facilitaría el desarrollo de su autonomía como sujeto cognoscente.

\section{CONCLUSIONES}

Este estudio sugiere una revisión curricular para visualizar obstáculos en la evaluación, coordinación entre docentes y dificultades para desarrollar la transposición didáctica de los contenidos disciplinarios. Asimismo, se evidenció deficiencias en la formación de los estudiantes, y por último, aparece el factor tiempo incidiendo en la calidad de la organización de la enseñanza. 
Es necesario considerar estos aspectos para tratarlos sistemáticamente en reuniones técnicas pedagógicas de la Carrera, consensuar criterios de acción en función de objetivos definidos por todos los actores involucrados, lo que implica la participación de los sujetos como estrategia de planificación.

Otro aspecto importante, está relacionado al análisis de los niveles de concepción de la realidad que poseen los docentes, al respecto es importante concluir desde estos tres criterios: ontológico, epistemológico y metodológico. En cuanto al criterio ontológico, los docentes conciben esencialmente la realidad como médicos, esto significa que la objetividad está presente como un elemento sustantivo al entender el proceso de enseñanza-aprendizaje, no considerando los aspectos dialécticos. Luego, es preciso reflexionar acerca del fondo epistemológico que cada docente confiere a sus prácticas, debido a que los resultados de este estudio revelan que existen incoherencias de carácter epistémico en el actuar pedagógico, que se reflejan en las estrategias de enseñanza aprendizaje que usan los docentes. Respecto de éstas es posible decir que se fundamentan en principios tradicionalistas de la enseñanza.

De lo anterior, el Espacio Europeo de Educación Superior (De Miguel) reconoce tácitamente los principios tradicionalistas de enseñanza, señalando la necesidad de un cambio de paradigma que apunta a que sea el propio alumno el que asuma la organización y desarrollo de su trabajo académico.

Esto se suma a las intenciones de aplicar procedimientos vinculados al paradigma constructivista del aprendizaje, no obstante esto debería reflejarse en la práctica docente, por ejemplo en la planificación. Respecto a esto De Miguel plantea que si la enseñanza está centrada en el alumno y las capacidades de éste, la planificación debería sufrir un cambio en esta dirección. Sin embargo, se aprecia una incongruencia entre la planificación y el "discurso constructivista" (Coll, 1993).

Finalmente, es importante discutir acerca de los su- puestos metodológicos, pues el actuar de los docentes está centrado más bien en lo experimental/tradicional, no considerando métodos dialógicos/dialécticos para aplicar en la enseñanza de la Anatomía. Por el contrario, el lenguaje usado en el aula apela a la exposición de conceptos, distanciándose de la construcción de éstos. En este sentido, no es posible realizar la transposición didáctica, pues al no estar presente el diálogo, la negociación y elaboración conjunta de significados, se tiende a omitir los aportes de los estudiantes. Para realizar la transposición didáctica del conocimiento, no sólo es importante el conocimiento disciplinario, sino también las particularidades de la cultura escolar (Díaz), esto significa no alejarse del conocimiento de los estudiantes, con lo que se lograría una adecuada transposición didáctica del conocimiento.

Es necesario tener los espacios necesarios de diálogo entre docentes, y cuestionarse la proporción de estudiantes por cada docente, que interviene en un adecuado desempeño docente. Pero, lo más importante es la coordinación entre los actores implicados en el proceso de enseñanza aprendizaje.

Se proyecta aportar a la reflexión en torno a la profesionalización del docente de Anatomía, en el sentido de promover un cambio de paradigma que estipula que el erudito es buen docente, a otro que plantea que el buen profesor se apoya en metodologías que permiten la transposición didáctica del conocimiento. Luego, diseñar planificaciones que expresen un modelo claramente definido y que dé cuenta de la transposición didáctica del conocimiento, esto guiado por un docente formado en ciencias de la educación.

Por tanto, se plantea la incorporación de los hallazgos del estudio en reuniones de coordinación docente y en otras instancias formales de diálogo en torno al proceso de enseñanza-aprendizaje. Por último, es interesante profundizar en las percepciones del estudiante respecto de la práctica docente en la Asignatura de Anatomía.

PELLÓN, A. M.; MANSILLA, S. J. \& SAN MARTíN, C. D. Challenges to the didactic transposition and knowledge of the content in teaching of anatomy: obstacles and projections. Int. J. Morphol., 27(3):743-750, 2009.

SUMMARY: Teaching undergraduate level anatomy classes demands a "formation" based in competences that provides learning results, where both "saberes sabios" and "saberes didacticos" converge. For that purpose, a qualitative design of research was proposed with the intent of describing requirements and characteristics of the teaching of anatomy courses in the medical school at the Universidad Mayor in Temuco, based on "grounded theory", "Constant Comparative Method" and "Methodological Triangulation". The sampling was composed of the entire teaching staff for the anatomy courses. As a result of open coding five categories were identified, and for this article the most representative one has been chosen: "requirements and characteristics of the teaching of anatomy". The techniques used for gathering information were "ethnographic observation", "structured interview" and focus groups. Data"reduction" was done with help of software for qualitative data analysis called "Atlas-ti" v5.0. The results of the study reveal incoherencies of "epistemic" character in the "didactic performance" of the teaching staff, who base their practice from the experimental logic, staying away of methods "dialogicos dialecticos" needed in the current sociocultural "cartography" shown by undergraduate level students.

KEY WORDS: Didactic transposition; Teaching of Anatomy; Disciplinary knowledge; Qualitative research. 
PELLÓN, A. M.; MANSILLA, S. J. \& SAN MARTíN, C. D. Desafíos para la transposición didáctica y conocimiento didáctico del contenido en docentes de anatomía: obstáculos y proyecciones. Int. J. Morphol., 27(3):743-750, 2009.

\section{REFERENCIAS BIBLIOGRÁFICAS}

Acevedo, J. Conocimiento didáctico del contenido para la enseñanza (I): El marco teórico. Revista Eureka sobre Enseñanza y Divulgación de las Ciencias, 6(1):41-6, 2009.

Coll, C. El constructivismo en el aula. Barcelona, Graó, 1993.

Chevallard, Y. La transposición didáctica. Del saber sabido al Saber enseñado. $1^{\mathrm{a}}$ Ed. Buenos Aires, Aique, 1985.

De Miguel-Díaz, M. Cambio de paradigma metodológico en la Educación Superior. Exigencias que conlleva. Cuadernos de Integración Europea, 2:16-27, 2005.

Díaz, T. La Interpretación Histórico-Cultural de la Transposición Didáctica como Puente de Emancipación del Aprendizaje y de la Enseñanza. Revista Praxis, 3:3756, 2003.

Flick, U. Introducción a la investigación cualitativa. Madrid, Morata, 2004.

Grossman, P.; Wilson, S. \& Shulman, L. Profesores de sustancia: el conocimiento de la materia para la enseñanza. Rev. Revista de currículum y formación del profesorado, 9:2-25, 2005.

Khun, T. La Estructura de las Revoluciones Científicas. México D.F., Fondo de Cultura Económica, 1982.

Shulman, L. Conocimiento y enseñanza: Fundamentos de la nueva reforma. Revista de currículum y formación del profesorado, 9(2):1-31, 2005.

Strauss, A. \& Corbin, J. Bases de la investigación cualitativa. Técnicas y procedimientos para desarrollar la teoría fundamentada. Medellín-Colombia, Universidad de Antioquia, 2002.
Dirección para correspondencia:

Mario Pellón Arcaya

Médico Cirujano Oftalmólogo

Magíster en Pedagogía Universitaria

Docente Facultad de Medicina

Universidad de La Frontera

Casilla 54-D.

Temuco

CHILE

Email: mpellon@ufro.cl

Recibido : 19-06-2009

Aceptado: 21-07-2009 